\title{
ESTUDO FÍSICO-QUÍMICO E REOLÓGICO DA POLPA DE MURICI
}

\author{
V.P. PIRES ${ }^{1}$, S. BORGES ${ }^{1}$, A.A. SANTANA ${ }^{1}$, R.M. SAMPAIO ${ }^{1}$, H.A. VILLA-VÉLEZ ${ }^{1}$ \\ ${ }^{1}$ Universidade Federal do Maranhão, Coordenação de Engenharia Química \\ E-mail para contato: valkerline.pires@hotmail.com
}

\begin{abstract}
RESUMO - O murici (Byrsonima crassifólia L. Rich) é um fruto oriundo da Amazônia Brasileira, estando presente em todo norte, nordeste e no cerrado Brasileiro. Seu fruto possui um alto teor de vitaminas e antioxidantes sendo sua polpa de grande procura no mercado. A polpa é comercializada como fruto in natura ou como polpa congelada, sendo está última feita de forma artesanal e micro-industrial. Poucos estudos sobre as propriedades físicas e reológicas da polpa de murici foram realizados. Assim, o presente trabalho tem como objetivo geral, estudar as propriedades reológicas e químicas da fruta de murici in natura a diferentes condições experimentais. Para isto, foi realizado um estudo reológico da polpa de murici a diferentes condições de temperatura e taxas de deformação empregando um viscosímetro. Outros estudos de análise físico-química foram realizados de acordo com os padrões de análise internacionais. As polpas apresentam comportamento não newtoniano e o ajuste foi feito através do modelo Lei da potência.
\end{abstract}

\section{INTRODUÇÃO}

O murici (Byrsonima crassifólia L. Rich), é um fruto oriundo da Amazônia Brasileira e Nordeste, mas pode ser cultivado no cerrado e sudeste. A planta é conhecida como muricizeiro e apresenta um formato de arbusto de 2 a $6 \mathrm{~m}$ de altura com ramos longos e casca espessa. $O$ seu fruto por sua vez exibe formas arredondadas de 1,5 a $2 \mathrm{~cm}$ de diâmetros com o exocarpo de coloração amarela e o mesocarpo de textura pastosa e cheiro característico (EMBRAPA, 2005).

A indústria de frutas no Brasil está em continuo crescimento oferecendo a cada dia produtos in natura com alto conteúdo nutricional a fim de atender a grande demanda do setor. No entanto, os referidos alimentos têm uma vida de prateleira curta, fazendo com que tecnologias de conservação ou processos mínimos sejam realizadas nas polpas de frutas (COSTA; SILVA et. al 2013). Na indústria de alimentos, o processamento de polpas de frutas é realizado controlando fatores como a temperatura e as propriedades físicas do produto, com a finalidade de evitar a degradação dos micro e macro nutrientes. Um dos métodos mais empregados para a análise e caracterização das frutas é baseado no seu comportamento reológico (EVANGELISTA; VIEITES, 2006). Segundo Ferreira et al., (2002), o comportamento reológico é definido como a deformação mecânica nos materiais causados por uma tensão. A reologia em alimentos é considerada uma ferramenta analítica que permite uma clara compreensão da organização estrutural dos materiais muito útil para a área da engenharia química fornecendo dados para a construção e projeção dos mais 
variados equipamentos (STEFFE, 1996). O conhecimento das características reológicas das polpas de frutas é de grande importância para se desenvolver novos produtos, porém fatores como temperatura, concentração e sólidos solúveis interferem na reologia das polpas e derivados (RAO, 1986).

Com base no argumento anterior, o presente trabalho tem como objetivo geral determinar as características reológicas e físicas da polpa de murici em função da taxa de deformação e temperaturas.

\section{MATERIAIS E MÉTODOS}

\subsection{Materiais}

$\mathrm{Na}$ pesquisa foi utilizado fruto de murici adquirido no mercado local. O produto foi selecionado nas concentrações de sólidos solúveis de 3, 5 e $10{ }^{\circ}$ Brix. Os frutos foram despolpados e armazenados em pacotes plásticos de polietileno e conservados freezer.

\subsection{Caracterização físico-química}

As polpas in natura de murici foram caracterizadas quimicamente de acordo com o "Official Methods of Analysis of AOAC INTERNATIONAL (AOAC, 2006) e com a metodologia do Instituto Adolfo Lutz (IAL, 2008)". Todos os ensaios foram feitos em triplicata.

\subsection{Estudo reológico e análise matemática}

Foi estudado o comportamento reológico das polpas de murici $\left(3,5\right.$ e $\left.6{ }^{\circ} \mathrm{Brix}\right)$ nas temperaturas de 10,35 e $60^{\circ} \mathrm{C}$ ultilizando um reômetro Brookfield, (modelo DV-II+Pro, Brookfield, USA). E um banho termostático a fim de controlar a temperatura das amostras.Cada análise reológica foi executada em triplicata, nas taxas de deformações 0,333 a $3,333 \mathrm{~s}^{-1}$. Onde dos dados foram ajustados com o modelo reológico de Ostwald-de-Waele, conforme a equação 1 . Além disso, através deste modelo foi estudado o efeito da temperatura na concentração de sólidos através da relação de do tipo Arrhenius, como mostrado na Eq. (2)

$$
\begin{gathered}
\mu=k \dot{\gamma}^{\eta-1} \\
\eta=A_{0} \exp \left(\frac{E_{a}}{R(T+273.15)}\right)
\end{gathered}
$$

Para a modelagem e simulação foi avaliado o grau de ajuste do modelo aos dados experimentais em função da magnitude do coeficiente de determinação ajustado $\left(R^{2}\right)$ e do erro médio estimado (MRE) (\%) (Eq. (3)).

$$
M R E=\frac{100}{N} \sum_{i=1}^{N} \frac{\left|X_{i}-X_{i}^{*}\right|}{X_{i}}
$$


Em que: $X_{i}$ é o valor observado experimentalmente; $X_{i}^{*}$ é o valor calculado pelo modelo e $N$ é o número de valores experimentais.

\section{RESULTADOS}

\subsection{Análises físico-químicas}

Os resultados das análises físico-químicas da polpa de murici in natura são apresentados na Tabela 1.

Tabela 1 - Composição centesimal do murici.

\begin{tabular}{|c|c|}
\hline Característica Físico- Químicos & Valores \\
\hline Umidade $(\mathrm{g} / 100 \mathrm{~g})$ & $88,22 \pm 0,14$ \\
\hline Cinzas $(\mathrm{g} / 100 \mathrm{~g})$ & $0,30 \pm 0,04$ \\
\hline Lipídeos & $1,45 \pm 0,07$ \\
\hline Proteínas & $0,86 \pm 0,08$ \\
\hline Carboidratos & $9,18 \pm 0,15$ \\
\hline Sólidos solúveis $\left(\mathrm{B}^{\circ}\right)$ & $5,93 \pm 0,06$ \\
\hline Sólidos totais & $11,78 \pm 0,15$ \\
\hline Acidez titulada em ácido cítrico & $0,94 \pm 0$ \\
\hline Vitamina C & $3,53 \pm 0,08$ \\
\hline Ph & $3,44 \pm 0,01$ \\
\hline
\end{tabular}

$\mathrm{O}$ valor da umidade encontrado neste trabalho para umidade foi semelhante aos atribuídos pelo IBGE (IBGE, 1999) de 82,8\%, apresentando também valores próximos aos dados obtidos por Silva (2008) que relataram valores de e $80,64 \%$ respectivamente. Com relação ao conteúdo de cinzas, foi obtido o valor de 0,29 , sendo este menor ao reportado por Monteiro (2015) de 0,69. A composição de ésteres encontrada foi de 1,45\%, sendo similares aos valores apresentados por Monteiro (2015). A amostra de polpa de murici no presente estudo apresentou dados experimentais de sólidos solúveis baixos e ácidos titulada em ácido cítrico alto. Quanto ao $\mathrm{pH}$, foram obtidos valores similares aos reportados por Monteiro (2015. Os frutos que apresentam caráter mais ácido são usados para produção de sorvete, sucos, doces. Quanto a quantidade de carboidratos presentes na amostra de murici usada, o valor de $9,17 \%$ é menor que os encontrados na literatura.

\subsection{Estudos Reológicos}

A Figura 1 mostra os resultados experimentais da viscosidade aparente no teor de sólidos solúveis de 3,5 e $6{ }^{\circ}$ Brix.

Como se pode ser vista na Figura 1 , a viscosidade aparente $\left(\mu_{\text {aparente }}\right)$ diminui à medida que a taxa de cisalhamento aumenta. Além disso, a viscosidade aparente também depende da temperatura, onde se pode ver claramente que as viscosidades diminuem à medida que a temperatura aumenta. Este decréscimo significa que a resistência das amostras ao escoamento e a energia requerida para mantê-los a uma alta taxa de deformação é reduzida. O mesmo comportamento foi descrito por Ferrreira et al., (2002) em estudo reológico de polpas de caju e goiaba. 
Figura 1 - Resultados experimentais da viscosidade aparente nas concentrações de $3{ }^{\circ} \mathrm{Brix}(\mathbf{\square}), 5^{\circ} \mathrm{Brix}(\boldsymbol{\Delta})$ e $6{ }^{\circ}$ Brix (•) para a temperatura e $60^{\circ} \mathrm{C}$ em função da taxa de deformação. Linhas (---) indicam a modelagem das curvas através do modelo Lei da Potência.

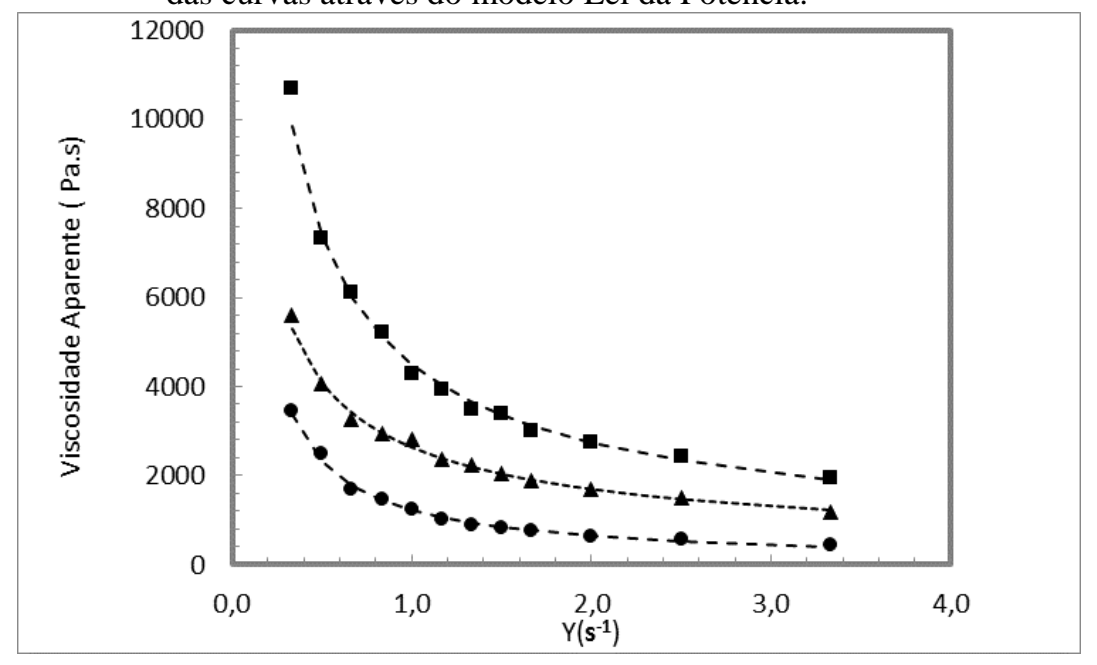

Na Tabela 2, observa-se que os valores de $R^{2}$ foram acima de 0,907 e os valores de $M R E$ menores que $10 \%$, mostrando que os dados experimentais de ajustam adequadamente ao modelo Lei da Potência. Por outra parte, os parâmetros de $k$ e $n$, em geral, diminuem a medida que aumenta a temperatura, sendo este comportamento inversamente proporcional. A temperatura é um dos fatores que mais afetam a viscosidade das polpas de murici, isso se dá pelo fato desses fluidos estarem formados basicamente de sólidos na presença de um líquido. O decréscimo da viscosidade ocorre quando a temperatura aumenta, pois a viscosidade do liquido presente na mistura aumenta e isso causa um aumento na movimentação de todo o sistema liquido-sólido, (PELEGRINE; VIDAL; GASPARETTO, 2000; KARWOWSKI, 2012).

Tabela 2 - Parâmetros do modelo de Ostwald-de-Waelle.

\begin{tabular}{|c|c|c|c|c|c|}
\hline${ }^{\mathbf{0}}$ Brix & Temperatura $\left({ }^{\circ} \mathbf{C}\right)$ & $\boldsymbol{k}$ (Pa.s) & $\boldsymbol{\eta}$ & $\boldsymbol{R}^{\mathbf{2}}$ & MRE (\%) \\
\hline \multirow{3}{*}{3} & 10 & 661,476 & 0,091 & 0,973 & 6,786 \\
\cline { 2 - 6 } & 35 & 277,0 & 0,356 & 0,970 & 5,896 \\
\cline { 2 - 6 } & 60 & 456,2 & 0,205 & 0,975 & 5,800 \\
\hline \multirow{3}{*}{5} & 10 & 1231,852 & 0,083 & 0,995 & 3,648 \\
\cline { 2 - 6 } & 35 & 4501,598 & 0,288 & 0,994 & 2,824 \\
\hline \multirow{3}{*}{6} & 60 & 2636,649 & 0,363 & 0,993 & 2,268 \\
\cline { 2 - 6 } & 10 & 661,996 & 0,571 & 0,907 & 9,943 \\
\cline { 2 - 6 } & 35 & 441,468 & 0,316 & 0,976 & 6,918 \\
\hline
\end{tabular}

$\mathrm{Na}$ polpa de murici a viscosidade aparente diminuiu conforme a temperatura aumentava. Esse comportamento é característico dos fluidos pseudospláticos, onde a viscosidade aparente diminui à medida que a taxa de cisalhamento. Nos fluidos alimentícios o comportamento da viscosidade aparente em relação ao aumento da temperatura, pode ser relacionado usando a equação de Arrhenius, Eq. (2). Onde a viscosidade aparente diminui em função exponencial com o aumento da temperatura (STEFFE, 1996). 
A Figura 2 mostra o comportamento da energia de ativação.

FIGURA 2 - Logaritmo natural do índice de comportamento em função do inverso da temperatura nas concentrações de $3{ }^{\circ} \operatorname{Brix}(\boldsymbol{\Delta}), 5^{\circ} \operatorname{Brix}(\bullet)$ e $6{ }^{\circ} \operatorname{Brix}(\boldsymbol{\bullet})$, respectivamente.

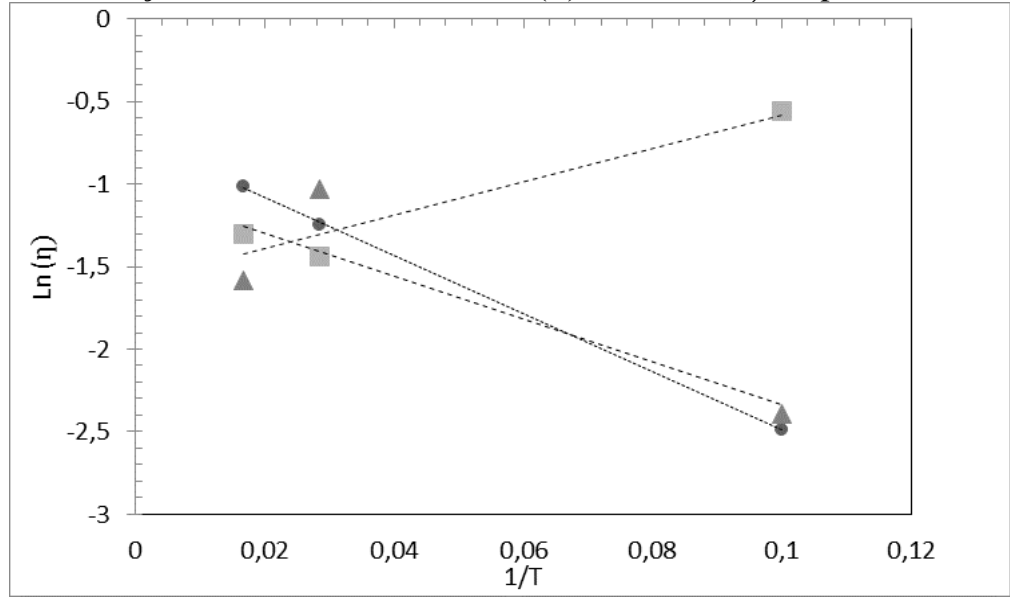

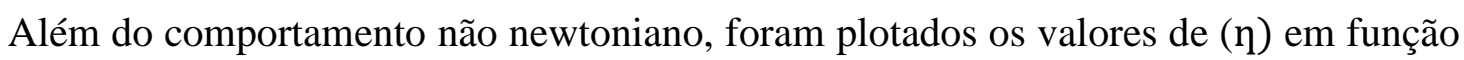
do inverso da temperatura para obter os valores da Energia de Ativação. Assim, através da regressão linear do modelo, foram obtidos os valores de $\left(E_{a}=3,36 ;-146 ; 11869,12 \mathrm{~J} / \mathrm{mol} \mathrm{K}\right)$ e o parâmetro de ajuste $\left(A_{0}=-2,57 ;-0,73 ;-5,66\right)$ para as concentrações de 3,5 e $6{ }^{\circ}$ Brix respectivamente. Segundo a literatura a energia de ativação é a energia necessária para que uma reação ocorra. Para a reologia, a energia de ativação afeta na viscosidade aparente do fluido através do aumento ou diminuição da temperatura. Então havendo um aumento da temperatura a energia de ativação também aumenta e, portanto a viscosidade aparente do fluido diminui o escoamento tende a ser mais rápido (PEREIRA; QUEIROZ; FIGUEIREDO, 2003).

\section{CONCLUSÕES}

Neste trabalho permitiu-se observa as características reológicas e propriedade térmicas da polpa do murici. Atrás dos resultados obtidos podem realizados estudos de modelagem e simulação na área das operações de escoamento e transferência de calor. De todos os modelos testados nesse trabalho, a lei da potência foi o que melhor descreveu o comportamento reológico do murici, por apresentar valores de $R^{2}$ mais próximos de 1 . As polpas de murici apresentaram uma característica de fluido não newtoniano em especial uma característica pseudoplática. Estudos de sedimentação e turbidez mostraram que estas propriedades são dependentes diretamente da velocidade de homogeneização do equipamento. Finalmente, o modelo tipo Arrehenius foi representativo para avaliar o efeito da temperatura no comportamento reológico da polpa de murici.

\section{REFERÊNCIAS}

A.O.A.C. Official Methods of Analysis. $18^{\text {th }}$ ed. Association of Official Analytical Chemists, Gaithersburg, Maryland, 2006. 
COSTA, Denise Oliveira da; CARDOSO, Gildene Romao; SILVA, Geandra Morais Valério da. A evolução do setor produtivo e comercialização de polpa de fruta no brejo paraibano: estudo de caso na coaprodes. Salvador, 2013.

EMBRAPA; Murici ( L.) Rich.). Porto Velho, RO, Agosto, 2005. Disponivel em: http://www.cpafro.embrapa.br/media/arquivos/publicacoes/folder_murici.pdf.Acesso em: 15 de Julho, 2016.

EVAngelista, R.M.; VIEITES, R.L. Avaliação da Qualidade de Polpa de Goiaba Congelada, Comercializada na Cidade de São Paulo. Segurança Alimentar e Nutricional, Campinas, 2006.

FERREIRA, G. M. et al. Efeito da temperatura no comportamento reológico das polpas de caju e goiaba. Ciências Exatas e Naturais, v. 4, n. 2, p. 176-184, 2002.

INSTITUTO ADOLFO LUTZ. Normas Analíticas do Instituto Adolfo Lutz. Métodos Químicos e Físicos para Análise de Alimentos. 4ª ed. São Paulo: IAL, 2008, 1020p.

MONTEIRO, Debora et al, Caracterização Físico-química do fruto e da Geleia do Murici, 4f. Artigo, Nutrição, Universidade Federal de Tocantins, 2015.

KARWOWSKI, M. S. M, Estudo da estabilidade, comportamento reológico e dos compostos fenólicos de frutas da mata atlântica. Dissertação (Mestrado), Programa de PósGraduação em Tecnologia de Alimentos. Universidade Federal do Paraná, 2011.

PELEGRINE, D. H.; VIDAL, J. R. M. B.; GASPARETTO, C. A. Estudo da viscosidade aparente das polpas de manga (Keitt) e abacaxi (Pérola). Ciência Tecnologia de Alimentos, v.20, n.1, 2000. p.128-131.

PEREIRA, E.A.; QUEIROZ, A.J.M.; FIGUEIREDO, R.M.F. Comportamento reológico de mel da abelha urucu (Melípona scutellaris, L.). Revista Ciências Exatas e Naturais, [sn] n.2, v.5, 2003.

SILVA, M.RI. LACERDA, D.B.C. L.; SANTOS, G.G.; MARTINS, D.M.O. Caracterização química de frutos nativos do cerrado. Ciência Rural, Santa Maria, v.38, n.6, set, 2008. p.1790-1793

STEFFE, J.F. Rheological methods in food process engineering. 2. ed. Freeman Press., [s.n] 1996. p. 412

RAO, M.A. Rheological properties of fluid foods. In: RAO, M.A.: RIZVI, S.S.H. (eds), Engineering propertires of foods. New York: Marcel Dekker, p. 1-47, 1986 